\title{
Adaptive Real-Time Estimation on Road Disturbances Properties Considering Load Variation via Vehicle Vertical Dynamics
}

\author{
Wuhui Yu, ${ }^{1}$ Xinjie Zhang, ${ }^{1,2,3}$ Konghui Guo, ${ }^{1,2}$ Hamid Reza Karimi, ${ }^{4}$ \\ Fangwu $\mathrm{Ma}^{3}{ }^{3}$ and Fumiao Zheng ${ }^{1}$ \\ ${ }^{1}$ State Key Laboratory of Automotive Simulation and Control, Jilin University, Changchun, Jilin 130022, China \\ ${ }^{2}$ State Key Laboratory of Advanced Design and Manufacture for Vehicle Body, Hunan University, Changsha, Hunan 410082, China \\ ${ }^{3}$ Zhejiang Geely Automobile Research Institute CO. L.t.d, Hangzhou 311228, China \\ ${ }^{4}$ Department of Engineering, Faculty of Engineering and Science, University of Agder, 4898 Grimstad, Norway
}

Correspondence should be addressed to Xinjie Zhang; xjzhang5885@gmail.com

Received 27 August 2013; Accepted 12 September 2013

Academic Editor: Hui Zhang

Copyright (C) 2013 Wuhui Yu et al. This is an open access article distributed under the Creative Commons Attribution License, which permits unrestricted use, distribution, and reproduction in any medium, provided the original work is properly cited.

\begin{abstract}
Vehicle dynamics are directly dependent on tire-road contact forces and torques which are themselves dependent on the wheels' load and tire-road friction characteristics. An acquisition of the road disturbance property is essential for the enhancement of vehicle suspension control systems. This paper focuses on designing an adaptive real-time road profile estimation observer considering load variation via vehicle vertical dynamics. Firstly, a road profile estimator based on a linear Kalman filter is proposed, which has great advantages on vehicle online control. Secondly, to minimize the estimation errors, an online identification system based on the Recursive Least-Squares Estimation is applied to estimate sprung mass, which is used to refresh the system matrix of the adaptive observer to improve the road estimation efficiency. Last, for mining road category from the estimated various road profile sequencse, a road categorizer considering road frequency and amplitude simultaneously is approached and its efficiency is validated via numerical simulations, in which the road condition is categorized into six special ranges, and this road detection strategy can provide the suspension control system with a better compromise for the vehicle ride comfort, handling, and safety performance.
\end{abstract}

\section{Introduction}

The road properties have significant impact on vehicle performance because vehicle dynamics are directly dependent on tire-road contact forces and torques. Road roughness is a broad term that incorporates everything from potholes and cracks to the random deviations that exist in a profile. The analysis and estimation of a road surface are hot and challenging topics, and the researches related to road disturbances have been presented, which can be classified into three categories: road measurement, road modeling, and road estimation. Each of these categories is reviewed briefly in the sequel.

Road Measurement. It mainly focuses on measuring the road profile accurately for road serviceability, survey, and road maintenance. There are primarily three methods in use internationally. The first category consists of profilometers and profilographs [1-4]. These are manually directed and/or trailer towed mechanisms that directly contact the pavement under evaluation, such as the longitudinal profile analyser (LPA). The second category is inertial profilers such as the Mays Meter [5] and the GMP (general motor profilometer) [6], in which a vehicle mounted accelerometer is applied to collect data while traveling at normal speed conditions. González et al. also developed a road roughness estimator by the use of acceleration measurement [7]. Furthermore, Ngwangwa et al. reconstructed road defects and road roughness classification using vehicle responses with artificial neural networks simulation [8]. The third category, equipped with a laser (ultrasonic) transceiver [9], is more accurate but very expensive. 
Road Modeling. It mainly focuses on providing road input source to the virtual vehicle simulation system or the FourPost Test Rig for vehicle ride analysis. In 1970s, the PSD (power spectral density) function was used by Whitehouse and Archard [10] and Shinozuka and Jan [11] to investigate the road roughness. In 1993, Cebon proposed a method based on the IFFT (inverse fast fourier transform) to discretize PSD, which is a simple, fast, and convenient tool for generating road surfaces $[12,13]$. In 1995, the contemporary international standard ISO 8608 [14] dealt with road roughness assumes, due to classification of roads into different classes according to their unevenness, equal intensity of road unevenness in the whole range of wavelengths and a general form of the fitted PSD were given. A method based on linear filtering (autoregressive and moving average method or ARMA modelling) was proposed by Yoshimura in 1998 [14], which has a smaller calculation, and a faster simulation speed, but its precision is not very well. Pazooki et al. [15], in 2007, summarized different stochastic models of parallel road tracks and evaluated their accuracy by comparing the difference of the measured parallel tracks and the synthetic parallel tracks. In 2012, Hassan and Evans [16] developed a comprehensive off-road vehicle ride dynamics model considering a random roughness model of the two parallel tracks.

Road Estimation. It mainly focuses on providing real-time rough road estimation for vehicle online control system. Since road input directly affects vehicle vertical suspension dynamics, the availability of suspension sensors such as accelerator and suspension deflection sensor provide an excellent opportunity for road input estimation. Fialho and Balas developed a road adaptive active suspension using linear parameter varying gain-scheduling [17], in which the road estimation results are applied to the suspension control system to achieve a better balance between ride and handling performance. A new simultaneous input and state estimation algorithm were developed based on the idea of achieving minimum mean square error and minimum error variance [18]. Two stable SISE algorithms were developed based on the minimum variance unbiased estimation technique [19]. A Takagi-Sugeno Fuzzy observer was built for estimating both vehicle dynamics and road geometry [20]. Vehicle sideslip and roll parameters are estimated in presence of the road bank angle and the road curvature as unknown inputs. But it is still not completely ready to be tested in experimental studies since the vehicle speed, parameters variations, and sensor noise are not considered. If the systems are subject to parameter uncertainties, the approaches proposed in [2123] can be employed. Our previous study [24, 25] also shows the great potential in the enhancement of suspension performance by adopting road estimation in the suspension control system, even though the road estimation algorithm is very simple (just using the statistic value of the accelerator or suspension deflection sensor measurement to classify the road). Recently, a real-time estimation method based on Kalman filter is proposed to estimate the road profile, and experimental results show the accuracy and the potential of the estimation process [26]. But in this research, the vehicle sprung mass change due to vehicle load variation is not considered, which may attenuate the robustness. Imine and Delanne developed a sliding mode observer to estimate the road profiles, which is hard for real-time implementation since a 16 degrees of freedom (DOF) full car model is too complex to be applied online $[27,28]$.

This paper focuses on developing a road estimation system for an online vehicle control system, which is limited by some important practical requirements. It should, for instance, be:

simple enough to run in real time despite onboard processing limitations;

reliable enough to operate successfully despite instrumentation failures;

robust to variations in vehicle dynamics;

fast enough to detect the road input changes when a car is driven on road;

easy to incorporate into a control strategy.

With these requirements in mind, the objective of this research has three steps: (1) to develop a road estimator for satisfying the above 5 practical requirements; (2) to obtain vehicle sprung mass online to minimize the road profile estimation errors; (3) and to classify the estimated road profile into several categories according to the main control strategy. The proposed method uses measurements from available sensors: accelerometers and suspension deflection sensors. For simplicity reasons, a quarter-car vehicle model is considered. The estimation process consisting of three blocks is shown in Figure 1.

The first block serves to calculate vehicle sprung mass online from sensor measurements, while the second block contains a Kalman filter that uses the result of the first block as a system parameter adjustor in order to improve robustness of the road estimation system. The third block serves to categorize the estimated road profile elevation into specific types.

The rest of the paper is organized as follows. Section 2 describes a road input state estimator based on a linear Kalman filter. Section 3 presents an online sprung mass estimator based on the Recursive Least-Squares Estimation. In Section 4, a novel road categorizer is proposed, and it is validated via numerical simulations. And, the paper is concluded in Section 5.

\section{Road Profile Estimator}

2.1. 2-DOF Quarter-Car Model. To implement the Kalman filter method, a suitable vehicle model must be developed. In order to describe the vertical dynamics of a vehicle which runs on an uneven road with a constant speed, a 2-DOF quarter-car model is represented in Figure 2. The quarter-car model does not consider the pitch and roll motions. Despite its simplicity, it captures the most basic feature of the vertical model of the vehicle [29]. We assume that wheels are rolling 


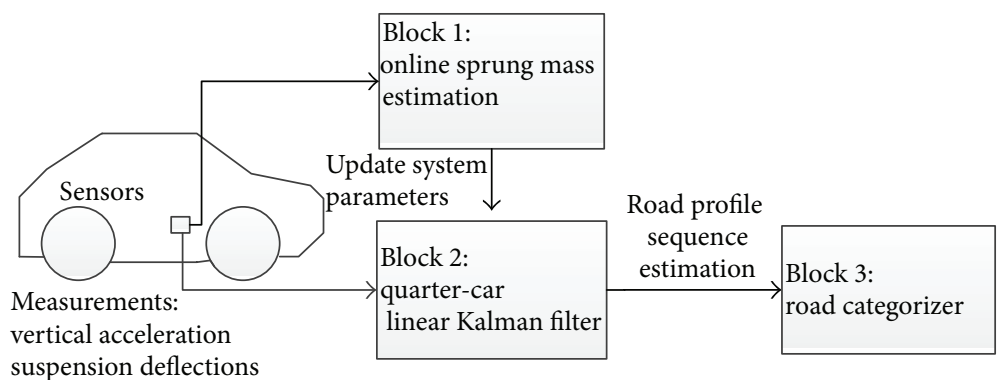

FIGURE 1: Road estimation process.

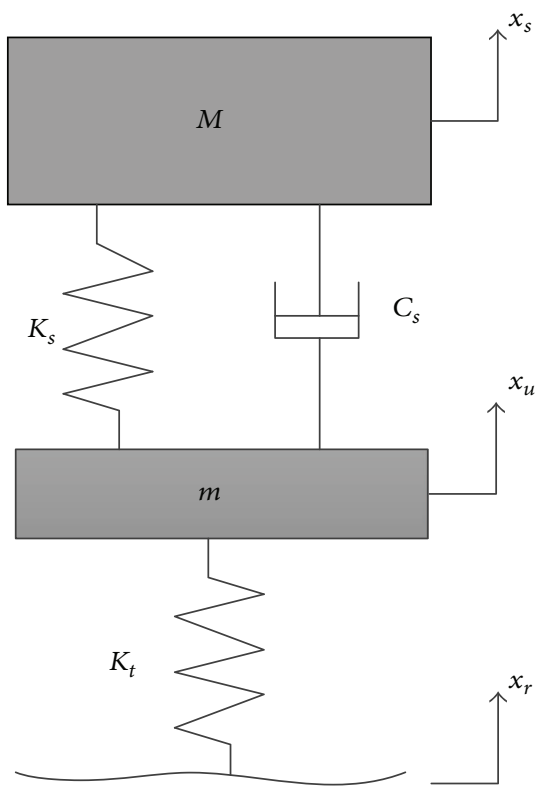

FIGURE 2: A quarter-car model.

without slip or contact loss. Equations (1) and (2) represent the vehicle body and the wheel motion, respectively:

$$
\begin{gathered}
m \ddot{x}_{u}-C_{s}\left(\dot{x}_{s}-\dot{x}_{u}\right)-K_{s}\left(x_{s}-x_{u}\right)+K_{t}\left(x_{u}-x_{r}\right)=0, \\
M \ddot{x}_{s}+C_{s}\left(\dot{x}_{s}-\dot{x}_{u}\right)+K_{s}\left(x_{s}-x_{u}\right)=0
\end{gathered}
$$

where $M$ and $m$ are, respectively, the sprung mass and the unsprung mass of a quarter car, $K_{s}$ represents the suspension stiffness, $K_{t}$ represents the tire vertical stiffness, $C_{s}$ is the damper damping coefficient, $x_{s}$ is the sprung mass position, $x_{u}$ is the unsprung mass position, and $x_{r}$ is the road input.

2.2. Kalman Filter for Road Input Estimation. The road input $x_{r}$ is an estimated signal and it should be a part of the system states. Hence, in this paper, state variables for the quarter car are presented as follows:

$$
\begin{array}{lll}
x_{1}=x_{s}, & x_{2}=\dot{x}_{s}, & x_{3}=x_{u}, \\
x_{4}=\dot{x}_{u}, & x_{5}=x_{r}, & x_{6}=\dot{x}_{r} .
\end{array}
$$

And the road roughness profile applied in the estimation observer satisfies the following equation [30]:

$$
\ddot{x}_{r}+a_{2} \dot{x}_{r}+a_{1} x_{r}=0,
$$

where $a_{1}$ and $a_{2}$ are constant real numbers. The modified quarter car has to be stable in order to provide useful result; this implies that the real parts of the eigenvalues associated with (4) have to be negative, and consequently $x_{r}$ converges to zero based on the selection of the constants $a_{1}$ and $a_{2}$. However, since $x_{r}$ represents the road profile input, it should contain as much road input information as possible. In other words, $x_{r}$ should be damped as slowly as possible, which will affect the stability of (4). Therefore, there is a tradeoff between system stability and road input information integrity. In this paper, $a_{1}$ and $a_{2}$ are set to 20 , which are close to two times of the quarter-car tire hop frequency, is sufficient to keep (4) stable [30]. The quarter car state equations can be written as follows:

$$
\begin{gathered}
x_{k+1}=A_{k} x_{k}+v_{k}, \\
y_{k}=H_{k} x_{k}+\omega_{k},
\end{gathered}
$$

where $x_{k}=\left(\begin{array}{llllll}x_{1, k} & x_{2, k} & x_{3, k} & x_{4, k} & x_{5, k} & x_{6, k}\end{array}\right)^{T}$ is a state vector; $y_{k}=\left(\left(x_{1, k}-x_{3, k}\right) x_{1, k} \quad \ddot{x}_{1, k}\right)^{T}$ is an observer vector, where $x_{1, k}-x_{3, k}$ is a suspension deflection measured by sensor; $x_{1, k}$ is a vehicle body position calculated by a twice numerical integration (trapezoidal method) of the filtered vertical acceleration signal; $\ddot{x}_{1, k}$ is a filtered vertical acceleration. $v_{k}$ and $\omega_{k}$ are the process and measurement noise vector, respectively, assumed to be white, zero mean, and uncorrelated.

Evolution and observation constant matrices are given as

$$
A=E+\Delta t\left(\begin{array}{ccccccc}
0 & 1 & 0 & 0 & 0 & 0 \\
-\frac{K t}{M} & -\frac{C s}{M} & \frac{K t}{M} & -\frac{C s}{M} & 0 & 0 \\
0 & 0 & 0 & 1 & 0 & 0 \\
\frac{K s}{m} & \frac{C s}{m} & -\frac{K s+K u}{m} & -\frac{C s}{m} & \frac{K u}{m} & 0 \\
0 & 0 & 0 & 0 & 0 & 1 \\
0 & 0 & 0 & 0 & -20 & -20 \\
1 & 0 & -1 & 0 & 0 & 0 \\
-\frac{K t}{M} & -\frac{C s}{M} & \frac{K t}{M} & \frac{C s}{M} & 0 & 0 \\
1 & 0 & 0 & 0 & 0 & 0
\end{array}\right),
$$


TABLE 1: Quarter-car model parameter.

\begin{tabular}{lcc}
\hline$\Delta t$ & Sampling period & $5 \mathrm{~ms}$ \\
$M$ & Vehicle sprung mass & $320 \mathrm{~kg}$ \\
$K_{s}$ & Suspension stiffness & $20.8 \mathrm{kN} / \mathrm{m}$ \\
$m$ & Unsprung mass & $40 \mathrm{~kg}$ \\
$K_{t}$ & Tire vertical stiffness & $235 \mathrm{kN} / \mathrm{m}$ \\
$C_{s}$ & Suspension damping & $1.5 \mathrm{kN} \cdot \mathrm{s} / \mathrm{m}$ \\
\hline
\end{tabular}

where $E$ is the identity matrix and $\Delta t$ is the sampling period. And it is easy to verify that the observability matrix $O$ is full rank:

$$
O=\left(\begin{array}{llllll}
H & H G & H G^{2} & H G^{3} & H G^{4} & H G^{5}
\end{array}\right)^{T} .
$$

A standard Kalman filter formulation is used for the velocity estimation $[31,32]$. The time update and measurement update equations of the filter are constructed as follows:

$$
\begin{gathered}
\text { time update } \\
\left\{\hat{x}^{-}\right\}_{k}=[A]\{\hat{x}\}_{k-1}+[B] u_{k-1}, \\
{\left[P^{-}\right]_{k}=[A][P]_{k-1}[A]^{T}+[Q],} \\
\text { measurement update } \\
{[K]_{k}=\left[P^{-}\right]_{k}[H]^{T}\left([H]\left[P^{-}\right]_{k}[H]^{T}+[R]\right)^{-1},} \\
\{\widehat{x}\}_{k}=\left\{\hat{x}^{-}\right\}_{k}+[K]_{k}\left(\{y\}_{k}-[H]\left\{\hat{x}^{-}\right\}_{k}\right), \\
{[P]_{k}=\left([I]-[K]_{k}[H]\right)\left[P^{-}\right]_{k},}
\end{gathered}
$$

where $Q$ is a process noise covariance matrix, $R$ is a measurement noise covariance matrix, $P$ is an estimation of error covariance matrix, $K$ is a Kalman gain, and $\widehat{x}$ is an estimated state vector. The process and measurement noise variables $v_{k}$ and $\omega_{k}$ determine how much should the process model and the measurements should be trusted by the filter.

2.3. Simulation Setup. This paper presents simulations of the quarter-car system as shown in Figure 2 with parameters tabulated in Table 1.

Two types of road inputs were used during experimental and simulation analysis. One input is a trigonal bump with $40 \mathrm{~mm}$ height and $400 \mathrm{~mm}$ length shown in Figure 3; another input is a random road input modeled based on the inverse Fourier transform as Figure 4.

Figure 5 to Figure 8 show a comparison between the actual and the estimated road profile height. In Figures 5 and 7 , the vehicle passes the bump at a constant speed of $15 \mathrm{~km} / \mathrm{h}$ and $45 \mathrm{~km} / \mathrm{h}$, respectively. In Figures 6 and 8 , the vehicle runs on a random road, and the speed is $30 \mathrm{~km} / \mathrm{h}$ and $60 \mathrm{~km} / \mathrm{h}$, respectively. The simulation results show that, in the random road input or the low speed bump input condition, the road estimator is very efficient, while in the condition of passing the bump with a relatively high speed, as shown in Figure 7, the estimation result is not very well. This is induced by the road model defined in (4). In fact, the road model applied in

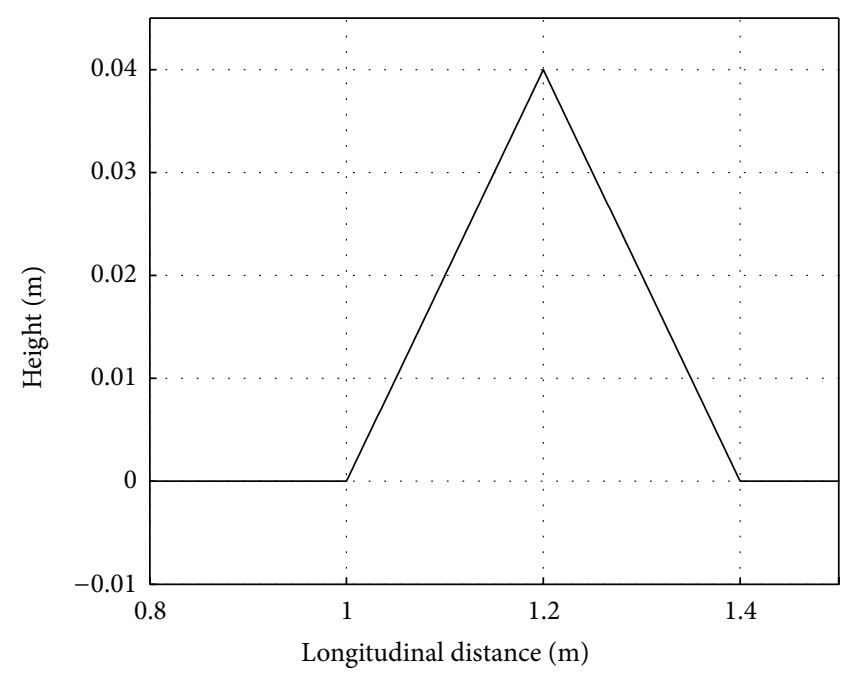

FIgURE 3: Bump road input.

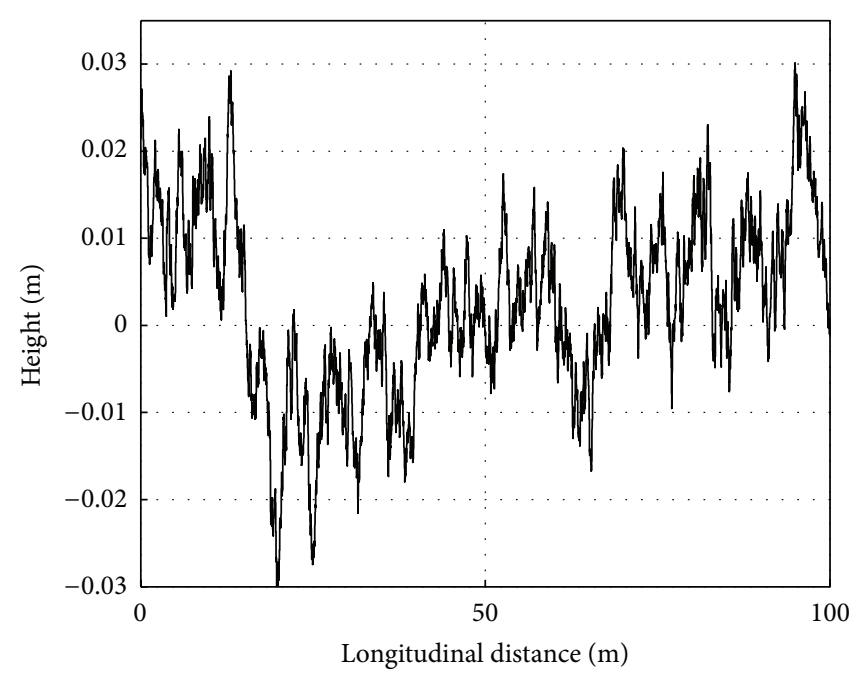

FIgURE 4: Random road input.

the proposed estimation observer should make a compromise between system stability and the integrity of road input information. We want to keep the system stable sacrificing the integrity of road input information in this research. And this strategy decreases the road estimator performance in an impulse input condition. The higher the vehicle speed is, the sharper the road input in time domain will be and the worse the road estimator will be. But its negative effect is acceptable since most drivers will reduce the vehicle speed when they run over a bump.

\section{Sprung Mass Estimation}

The road estimation method presented afore is based on a hypothesis that the vehicle system parameters in Table 1 are known and invariable. But in a real vehicle control system, it is not easy to obtain the sprung mass of the equivalent 2-DOF model, because of the nonlinear properties of the suspension system and the weight distribution variation on 


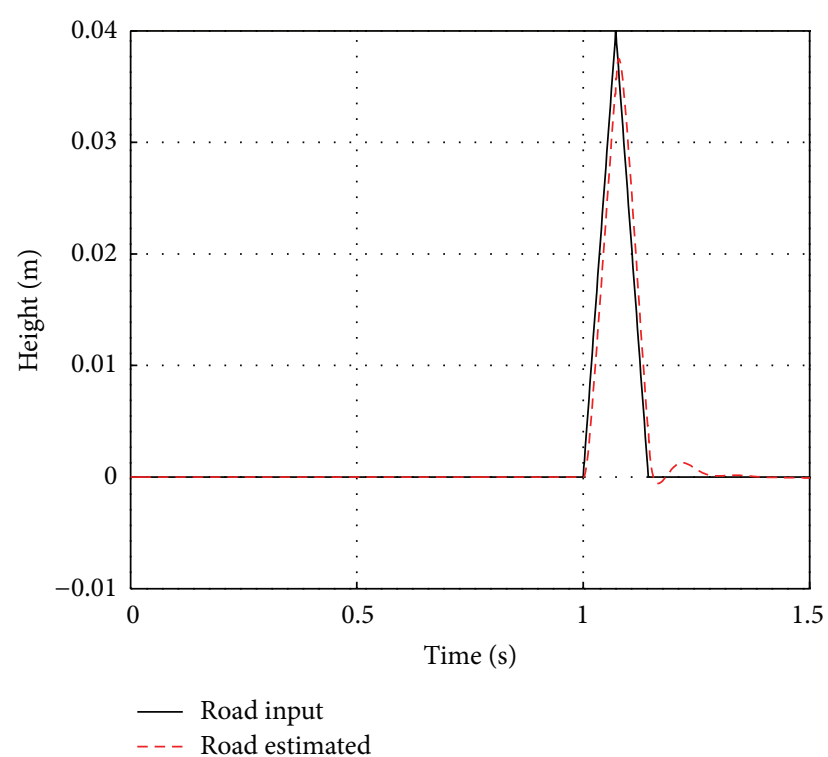

FIGURE 5: Bump input estimation $(15 \mathrm{~km} / \mathrm{h})$.

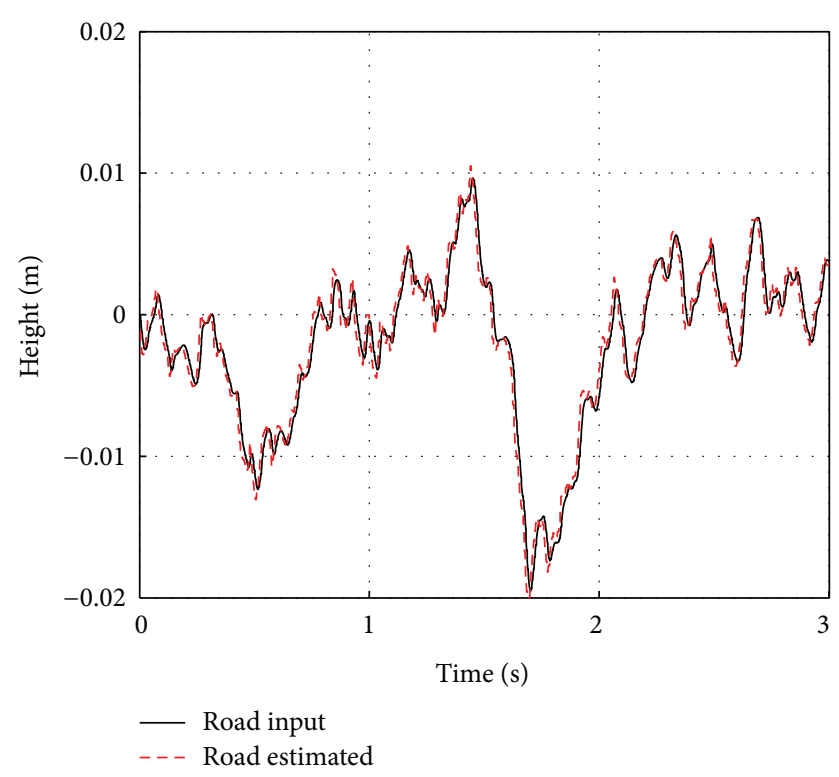

FIGURE 6: Random road estimation ( $30 \mathrm{~km} / \mathrm{h})$.

different driving conditions. What is more, the sprung mass varies greatly between empty and full loaded, especially for a commercial vehicle. All these factors will induce a significant sprung mass change and, then, attenuate the efficiency of the road estimation system. As shown in Figure 9, 20\% sprung mass error will induce an obvious road estimation error. Consequently, it is necessary to estimate the sprung mass online for improving the efficiency of road estimation.

3.1. Solution Formulation. With setting $y=-K_{s}\left(x_{s}-x_{u}\right), \theta=$ $\left[M, C_{s}\right]^{T}$, and $\psi^{T}=\left[\ddot{x}_{s}, \dot{x}_{s}-\dot{x}_{u}\right],(2)$ can be written as

$$
y=\psi^{T} \cdot \theta .
$$

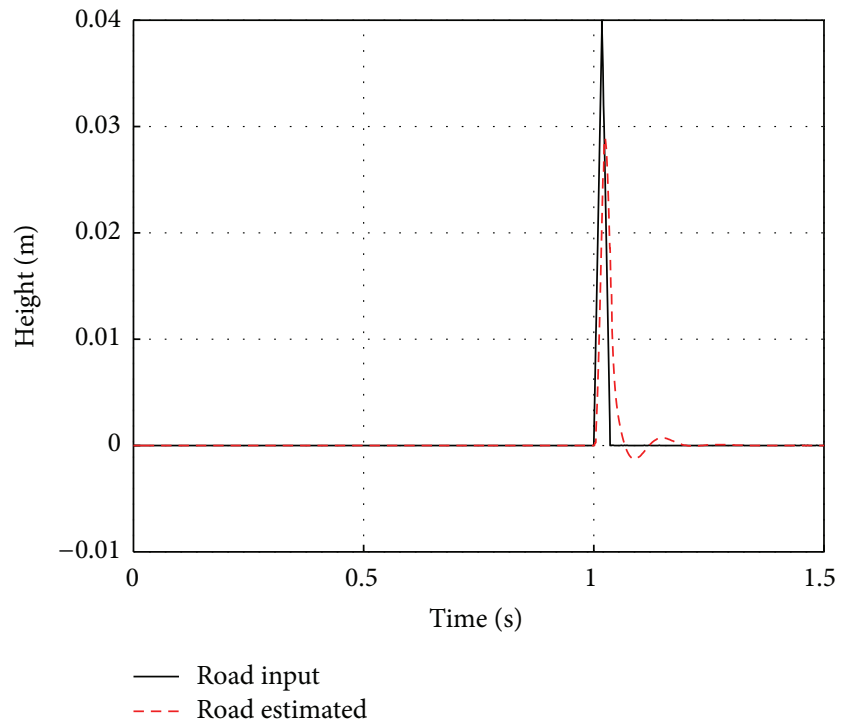

FIGURE 7: Bump input estimation (45 km/h).

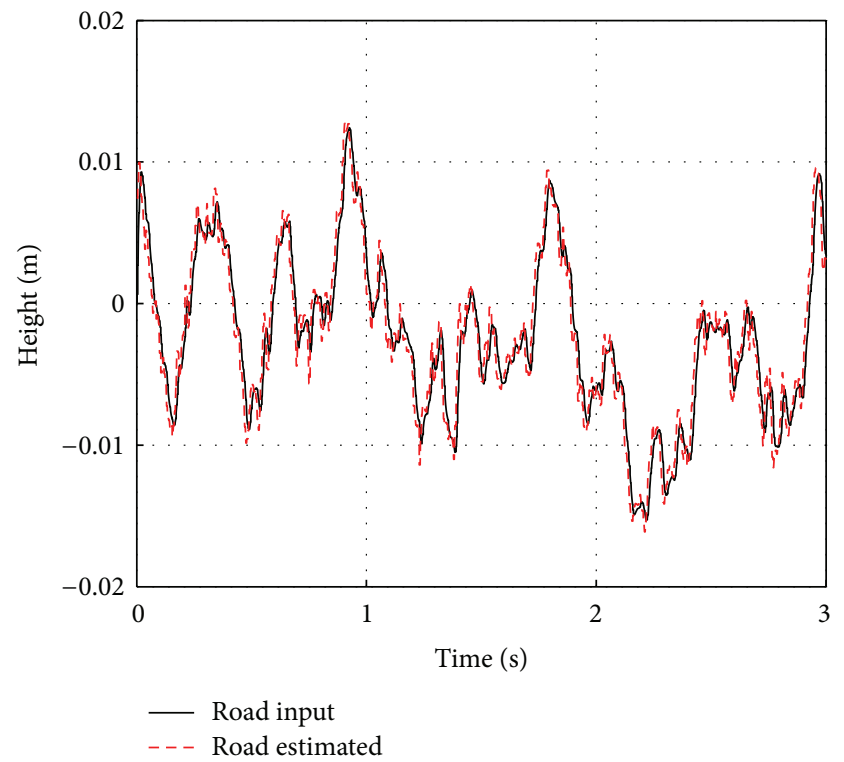

FIGURE 8: Random road estimation $(60 \mathrm{~km} / \mathrm{h})$.

The vehicle sprung mass and suspension damping coefficient are estimated simultaneously via the Recursive LeastSquares Estimation. The Recursive Least-Squares Estimation procedures are given as follows:

$$
\begin{gathered}
\widehat{\theta}_{N+1}=\widehat{\theta}_{N}+K_{N+1}\left(y_{N+1}-\psi_{N+1}^{T} \widehat{\theta}_{N}\right), \\
K_{N+1}=\frac{P_{N} \psi_{N+1}}{1+\psi_{N+1}^{T} P_{N} \psi_{N+1}}, \\
P_{N+1}=P_{N}-\frac{P_{N} \psi_{N+1} \psi_{N+1}^{T} P_{N}}{1+\psi_{N+1}^{T} P_{N} \psi_{N+1}} \\
=\left(I-K_{N+1} \psi_{N+1}^{T}\right) P_{N}
\end{gathered}
$$




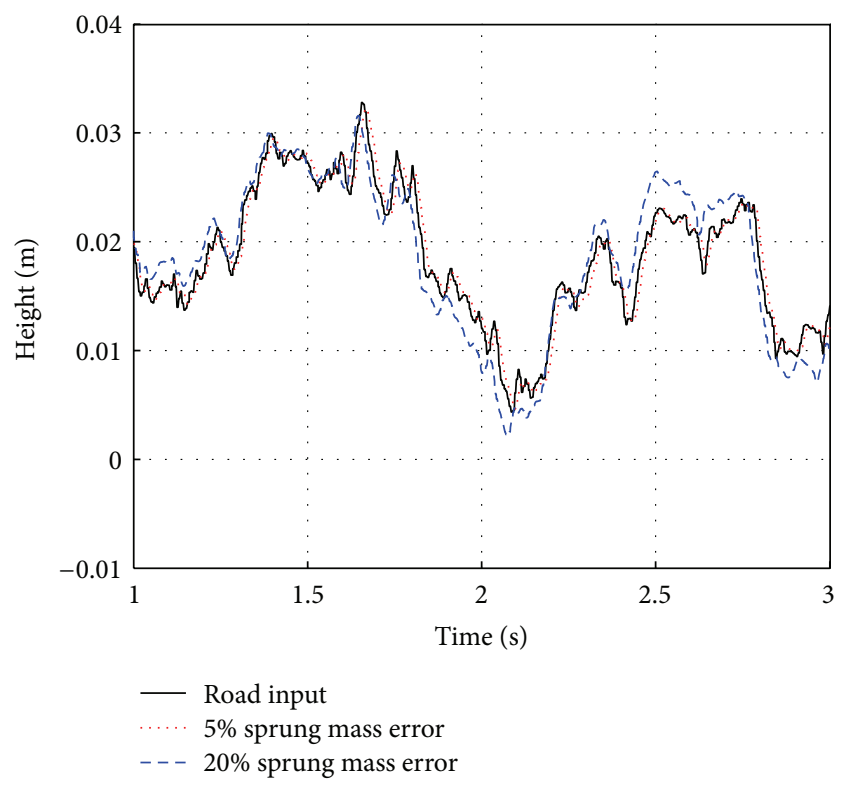

FIGURE 9: Road estimation on different sprung mass errors.

where $P$ is a symmetric covariance matrix $(2 \times 2)$ and $\widehat{\theta}_{N}$ is the Least-Squares estimation of $\theta$; the calculation order is described as follows.

Calculate $K_{1}$ by $\hat{\theta}_{0}$ and $P_{0}$ according to (11) first; then, update the value of $y_{1}$ and $\psi_{1}^{T}$; and then, calculate $\widehat{\theta}_{1}$ and $P_{1}$ according to (10) and (12), respectively; last, recursive the mentioned steps in order until the error reaches the standard. As for the initial values, $\widehat{\theta}_{0}$ and $P_{0}$, they can be calculated by the Least-Squares Estimation.

3.2. Simulation. Here, the vehicle system model parameters are from Table 1; the road profile defined in Figure 4 is selected as a vertical road input and the vehicle runs with a constant speed of $20 \mathrm{~km} / \mathrm{h}$. The estimation result is shown in Figure 10, which demonstrates the estimation result converges at an acceptable region (the error is less than $5 \%$ ) after $2.5 \mathrm{~s}$ and then shows great efficiency after $20 \mathrm{~s}$. Generally, the vehicle load is a constant during a vehicle start-stop period and the convergence time for sprung mass estimation is usually far less than a vehicle start-stop period. The sprung mass estimation is a little bit time-consuming, but it is not a big issue. For example, the original sprung mass is set in normal load condition. The road estimation block will be triggered once the vehicle has been started and the vehicle velocity is greater than $10 \mathrm{~km} / \mathrm{h}$; it will obtain the sprung mass (the error is less than $5 \%$ ) in $2.5 \mathrm{~s}$ after the road estimation block is triggered, and the sprung mass will be refreshed. And then the sprung mass will be updated in every $2.5 \mathrm{~s}$ until it is stable (the change is less than 1\%) or the estimation time is more than $40 \mathrm{~s}$.

\section{Road Categorizer}

In the previous sections, the road estimator performs very well in a quarter-car system. However, does this estimator

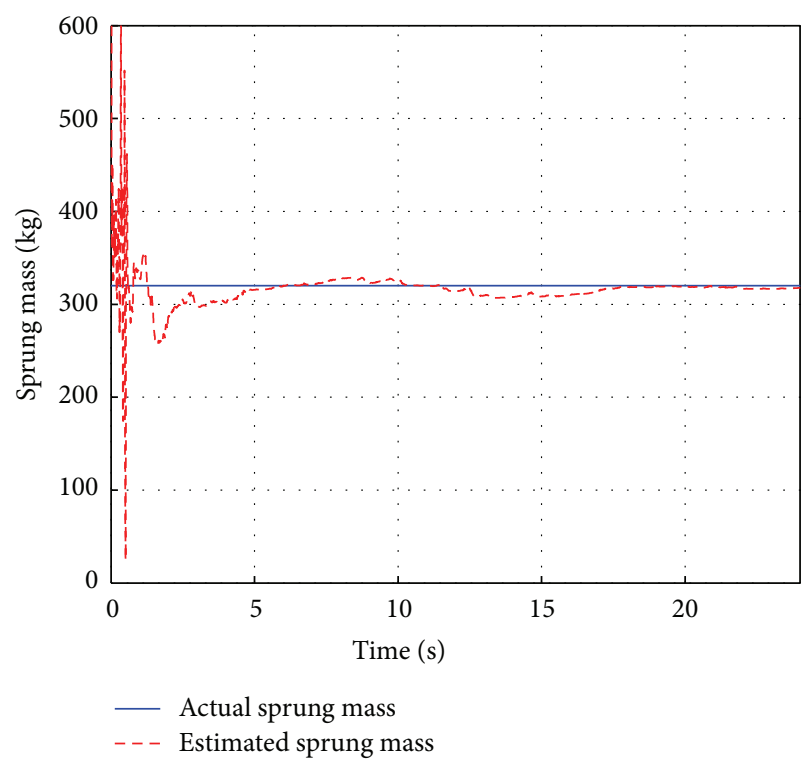

FIGURE 10: Sprung mass estimation.

have the same performance applied to an actual car online system? It cannot be denied that the estimated result, in an actual car online system, will be worse than expectation due to the effect of wheel radius, wheel contact area, and the noise signals in the control system. What is more, the estimated road profile cannot be applied to the control strategy directly since these road sequences are estimated by postestimation, which produces an unavoidable time delay. However, in many vehicle control systems, such as active/semiactive suspension system, obtaining the primary road category information is effective [33]. Hence, it is necessary to develop a road categorizer to distinguish the road category for the main control strategy. But the most common road classification methods, estimating the road PSD (power spectral density) or the road RMS (root mean square), are not enough effective for an online control system, because these methods need to compute plenty of road height values which will consume a lot of estimation time, and this time lag usually cannot be neglected in a real-time control system.

In fact, most onboard suspension control systems, only require the mainly frequency or amplitude information of road input. In $[33,34]$, a road-frequency adaptive suspension is proposed, where the road surface is classified by the frequency properties according to the fact that $[0-4 \mathrm{~Hz}]$ is the car-body frequency region and $[4-8 \mathrm{~Hz}]$ is the human-body frequency region. [8-12 Hz] is the wheel frequency region and $[12-\infty \mathrm{Hz}]$ is the harshness frequency region. Bastow et al. also categorize the road profile into four grades, which defined a very good surface with amplitudes under $5 \mathrm{~mm}$, medium-quality roads with amplitudes less than $13 \mathrm{~mm}$, poor-quality roads with amplitudes less than $25 \mathrm{~mm}$, and off-road with the amplitudes often exceeding $25 \mathrm{~mm}$ [35]. However, it is a challenging issue to design a proper filter to avoid some unimportant long waves without any phase delay, as shown in Figure 4; the road waves are not fluctuating along the horizontal plane due to the effect of some long waves 

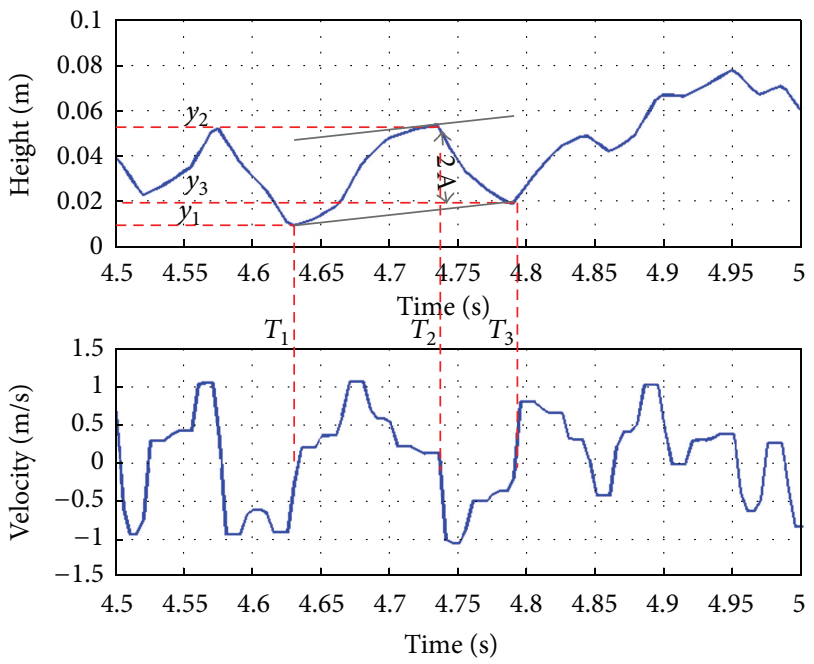

FIGURE 11: Road amplitude estimation method.

which has small contribution on the vehicle vertical dynamic; consequently, we should estimate the road input frequency and amplitude avoiding the unimportant long waves. In the other hand, the road categorizer only depending on the road frequency or the road amplitude does not work very well always. And the suspension control strategy should consider the road frequency and amplitude simultaneously in some case, such that a car runs on a road twice with different speed or a car runs on different road (ISO Class A and ISO Class C) with same speed. It will be much better if the road categorizer considers road frequency and amplitude simultaneously. Hence, a novel road categorizing method considering road frequency and amplitude simultaneously is proposed in the following paragraph.

For road frequency estimation, refer to the article [34], a first-order zero-crossing algorithm is applied, with the road velocity state $\dot{x}_{r}$ being the input, to identify the frequency components of road disturbances. The frequency estimation result of Figure 11 is shown in Figure 12, and the current estimation value is actually the last circle's frequency.

As for road amplitude analysis, road velocity state $\dot{x}_{r}$ does not contain the amplitude information, but the road amplitude estimation only via $x_{0}$ is very difficult, since some of the significant road waves are not fluctuating along the horizontal plane. As shown in Figure 11, the value of " $A$ " is one of the main amplitudes values, which is the amplitude of the wave with dominant frequency, should be estimated.

In the sequel, a novel road amplitude estimation method is proposed as shown in Figure 11, where, $T_{1}, T_{2}$, and $T_{3}$, used to detect each complete cycle, are the three attached times when first-order zero-crossing of the road velocity sequence happens; $y_{1}, y_{2}$, and $y_{3}$ are the three road height values in the times $T_{1}, T_{2}, T_{3}$, respectively. Then the road amplitude " $A$ " can be simply calculated as (13), where $T_{1}, T_{3}$ are used to detect the troughs of a circle and $T_{2}$ used to detect the peak of a circles are the distance between the point $\left(T_{2}, y_{2}\right)$ to the

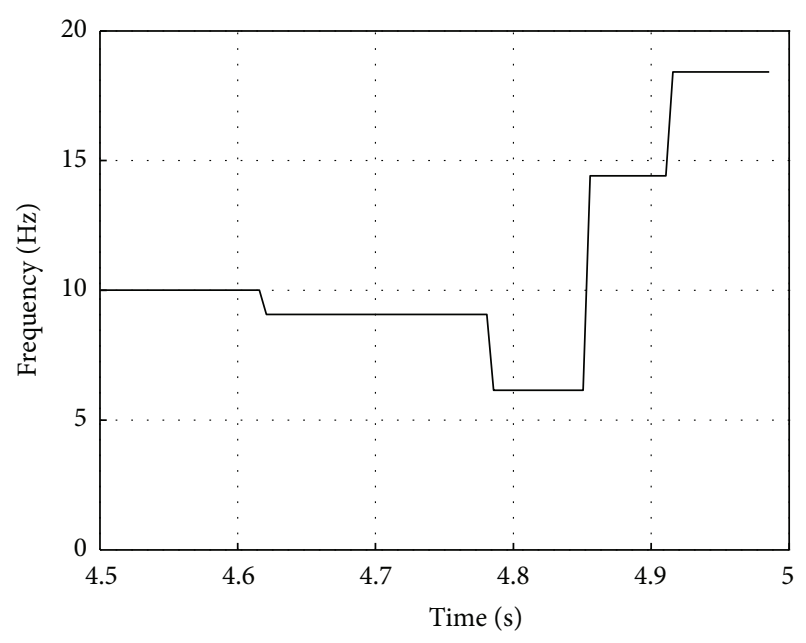

FIGURE 12: Road frequency estimation.

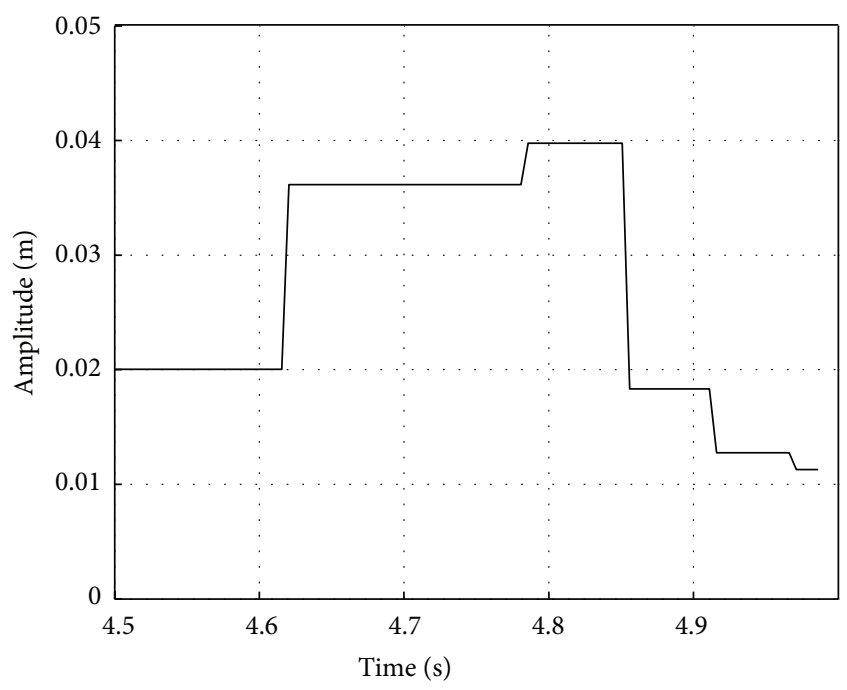

FIGURE 13: Road amplitude estimation.

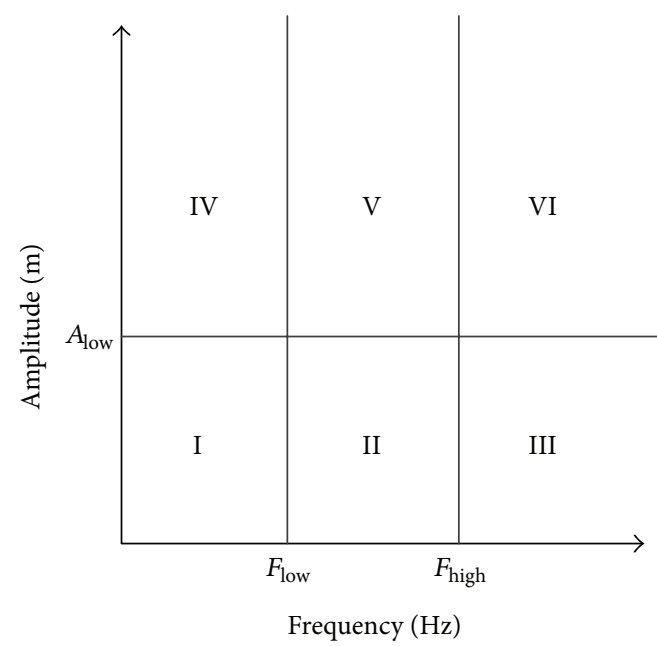

FIGURE 14: Road detection strategy. 
TABLE 2: Suspension mode selection based on road condition.

\begin{tabular}{lll}
\hline Range & Driving condition & Suspension control objective \\
\hline I and II & Good road surface with medium or low speed, mostly on an urban driving condition & Improve the vehicle ride performance \\
III & Expressway driving condition & Improve the road holding \\
IV & Bad road with low speed & Limit the low frequency body motion \\
V and VI & Poor-quality road surface with medium-high speed and the impulse road input & Attenuate the impact feeling \\
\hline
\end{tabular}

line going through the points $\left(T_{1}, y_{1}\right),\left(T_{3}, y_{3}\right)$; is two times of the amplitude $A$; in this paper, an approximate algorithm is used as follows:

$$
A= \begin{cases}\frac{1}{2}\left|y_{2}-\left(y_{1}+\frac{T_{2}-T_{1}}{T_{3}-T_{1}}\left(y_{3}-y_{1}\right)\right)\right| & \text { if } y_{3} \geq y_{1}, \\ \frac{1}{2}\left|y_{2}-\left(y_{3}+\frac{T_{3}-T_{2}}{T_{3}-T_{1}}\left(y_{1}-y_{3}\right)\right)\right| & \text { if } y_{3}<y_{1} .\end{cases}
$$

The amplitude estimation result is shown in Figure 13; even though the algorithm is running in real time, the estimation result still has a complete cycle time lag.

As road frequency and amplitude estimation can be achieved, a road categorizer special for a semiactive suspension control system can be designed, as shown in Figure 14. A certain type of road can be detected by judging the amplitude and frequency of the estimated road profile into the specific range (I-VI), where the suggestion values of $A_{\text {low }}$, $F_{\text {low }}$, and $F_{\text {high }}$ are $10 \mathrm{~mm}, 4 \mathrm{~Hz}$, and $8 \mathrm{~Hz}$, respectively. The suspension mode selection based on the road condition in specific range (I-VI) is detailed in Table 2. Ranges I and II are the medium-low frequency and low amplitude areas, corresponding to good road surface with medium-low speed, mostly on an urban driving condition. In this case, the suspension system should be turned to soft mode to improve the vehicle ride performance. Range III is the high frequency and low amplitude area, corresponding to the expressway driving condition, in which more attention should be paid to the tire deflection, and a relative hard mode suspension system should be regulated to achieve better road holding for driving safety. Range IV is the low frequency and high amplitude area corresponding to a bad road with low speed, which is very common for an off-road. In this condition, limiting the low frequency body motion should be a key objective. Ranges V and VI correspond to poor-quality road surface with medium-high speed and the impulse road input. In these cases, the suspension system should be adjusted to attenuate the impact feeling.

\section{Conclusions}

An acquisition of road disturbances property is essential for the enhancement of suspensions control systems. This paper presented a method to estimate the road profile elevation based on Kalman filter. To minimize the estimation errors, an online identification system based on Recursive LeastSquares Estimation is adopted to estimate sprung mass in real time, which is applied to refresh the system matrix of the adaptive observer. And a novel road categorizer considering road frequency and amplitude simultaneously is approached to classify various road profile sequence for suspension control system. The main conclusions are as follows.

(1) A road profile estimator based on linear Kalman filter is proposed, which has great advantages on practical online vehicle control.

(2) An online sprung mass estimator is proposed, which demonstrates the estimation result converges at an acceptable region (the error is less than 5\%) after $2.5 \mathrm{~s}$ and then shows great efficiency after $20 \mathrm{~s}$. With this online sprung mass estimator, the accuracy road estimation result can be improved greatly.

(3) A novel road amplitude estimation method is proposed. And the road condition is categorized into six special ranges according to the road frequency and amplitude estimation result simultaneously, which can provide the suspension control system with a better trade-off for the ride comfort, handling, and safety performance.

\section{Conflict of Interests}

The authors declare that there is no conflict of interests regarding the publication of this paper.

\section{Acknowledgments}

Special thanks are due to the Open Research Fund Program of the State Key Laboratory of Advanced Design and Manufacturing for Vehicle Body (31115028), the China Postdoctoral Science Foundation (2012M520028), the National Natural Science Foundation of China (51205155), and the National Basic Research Program of China (973 Program) (2011CB711201) for supporting authors' research.

\section{References}

[1] R. P. Barre, R. T. Forbes, and S. Andrews, "The measurement and analysis of road surface roughness," Motor Industry Research Association Report, 1970.

[2] M. W. Sayers, T. D. Gillespie, and W. D. O. Paterson, "Guidelines for conducting and calibrating road roughness measurements," World Bank Technical Paper Number 46, 1986.

[3] ISO 8608, Mechanical Vibration Road Surface Profiles Reporting of Measured Data, International Standards Organization, 1995.

[4] "Standard test Method for measuring road roughness by static rod and level method," Annual Book of ASTM Standards, vol. 4, pp. 750-755, 1996.

[5] "Standard test method for measuring longitudinal profile of traveled surfaces with an accelerometer: established inertial 
profiling Reference," Annual Book of ASTM Standards, no. 4, pp. 702-706, 1996.

[6] E. B. Splanger and W. J. Kelly, "Road profilometer method for measuring road profile," General Motors Research Publication GMR-452, 1964.

[7] A. González, E. J. O’Brien, Y.-Y. Li, and K. Cashell, “The use of vehicle acceleration measurements to estimate road roughness," Vehicle System Dynamics, vol. 46, no. 6, pp. 483-499, 2008.

[8] H. M. Ngwangwa, P. S. Heyns, F. J. J. Labuschagne, and G. K. Kululanga, "Reconstruction of road defects and road roughness classification using vehicle responses with artificial neural networks simulation," Journal of Terramechanics, vol. 47, no. 2, pp. 97-111, 2010

[9] R. McCann and S. Nguyen, "System identification for a modelbased observer of a road roughness profiler," in Proceedings of IEEE Region 5 Technical Conference (TPS '07), pp. 336-343, April 2007.

[10] D. J. Whitehouse and J. F. Archard, “The properties of random surfaces of significance in their contact," Proceedings of the Royal Society of London. Series A, vol. 316, pp. 97-121, 1970.

[11] M. Shinozuka and C.-M. Jan, "Digital simulation of random processes and its applications," Journal of Sound and Vibration, vol. 25, no. 1, pp. 111-128, 1972.

[12] D. Cebon, Interaction between Heavy Vehicles and Roads, Society of Automotive Engineers, 1993.

[13] J.-J. Wu, "Simulation of rough surfaces with FFT," Tribology International, vol. 33, no. 1, pp. 47-58, 2000.

[14] T. Yoshimura, "A semi-active suspension of passenger cars using fuzzy reasoning and the field testing," International Journal of Vehicle Design, vol. 19, no. 2, pp. 150-166, 1998.

[15] A. Pazooki, S. Rakheja, and D. Cao, "Modeling and validation of off-road vehicle ride dynamics," Mechanical Systems and Signal Processing, vol. 28, pp. 679-695, 2012.

[16] R. Hassan and R. Evans, "Road roughness characteristics in car and truck wheel tracks," International Journal of Pavement Engineering, pp. 1-10, 2012.

[17] I. Fialho and G. J. Balas, "Road adaptive active suspension design using linear parameter-varying gain-scheduling," IEEE Transactions on Control Systems Technology, vol. 10, no. 1, pp. 43-54, 2002.

[18] H. Fang, Y. Shi, and J. Yi, "A new algorithm for simultaneous input and state estimation," in Proceedings of the American Control Conference (ACC 08), pp. 2421-2426, Seattle, Wash, USA, June 2008.

[19] H. Fang, Y. Shi, and J. Yi, "On stable simultaneous input and state estimation for discrete-time linear systems," International Journal of Adaptive Control and Signal Processing, vol. 25, no. 8, pp. 671-686, 2011.

[20] H. Dahmani, M. Chadli, A. Rabhi, and A. El Hajjaji, "Vehicle dynamics and road geometry estimation using a Takagi-Sugeno fuzzy observer with unknown inputs," in Proceedings of IEEE Intelligent Vehicles Symposium (IV '11), pp. 272-277, BadenBaden, Germany, June 2011.

[21] H. Zhang, Y. Shi, and A. Saadat Mehr, "Robust static output feedback control and remote PID design for networked motor systems," IEEE Transactions on Industrial Electronics, vol. 58, no. 12, pp. 5396-5405, 2011.

[22] H. Zhang, Y. Shi, and A. S. Mehr, "Robust $H_{\infty}$ PID control for multivariable networked control systems with disturbance/noise attenuation," International Journal of Robust and Nonlinear Control, vol. 22, no. 2, pp. 183-204, 2012.
[23] H. Zhang, Y. Shi, A. Saadat Mehr, and H. Huang, "Robust FIR equalization for time-varying communication channels with intermittent observations via an LMI approach," Signal Processing, vol. 91, no. 7, pp. 1651-1658, 2011.

[24] K. H. Guo and W. H. Yu, "Semi-active suspension adaptive control strategy," Journal of Hunan University (Natural Sciences), no. 40, pp. 39-44, 2013.

[25] X. J. Zhang and W. H. Yu, "Semi-active suspension adaptive control strategy based on hybrid control," in FISITA World Automotive Congress, 2012.

[26] M. Doumiati, A. Victorino, A. Charara, and D. Lechner, "Estimation of road profile for vehicle dynamics motion: experimental validation," in Proceedings of the American Control Conference (ACC '11), pp. 5237-5242, San Francisco, Calif, USA, July 2011.

[27] H. Imine, Y. Delanne, and N. K. M'Sirdi, "Road profile input estimation in vehicle dynamics simulation," Vehicle System Dynamics, vol. 44, no. 4, pp. 285-303, 2006.

[28] H. Imine, Y. Delanne, and N. K. M'Sirdi, "Road profile inputs for evaluation of the loads on the wheels," Vehicle System Dynamics, vol. 43, pp. 359-369, 2005.

[29] M. M. Elmadany and Z. S. Abduljabbar, "Linear quadratic Gaussian control of a quarter-car suspension," Vehicle System Dynamics, vol. 32, no. 6, pp. 479-497, 1999.

[30] S. Nguyen, System Identification for a Model Based Observer Road Roughness Profiler, University of Arkansas, 2006.

[31] W. Greg and B. Gary, An Introduction to the Kalman Filter, Department of Computer Science, University of North California at Chapel Hill, 2006.

[32] R. E. Kalman, "A newapproach to linear filtering and prediction problems," Transactions of the ASME-Journal of Basic Engineering, no. 82, pp. 35-45, 1960.

[33] J. Lu, "A frequency-adaptive multi-objective suspension control strategy," Journal of Dynamic Systems, Measurement and Control, vol. 126, no. 3, pp. 700-707, 2004.

[34] L. H. Nguyen, K.-S. Hong, and S. Park, "Road-frequency adaptive control for semi-active suspension systems," International Journal of Control, Automation and Systems, vol. 8, no. 5, pp. 1029-1038, 2010.

[35] D. Bastow, G. Howard, and J. P. Whitehead, Car Suspension and Handling, SAE International, Warrendale, $\mathrm{Pa}$, USA, 4th edition, 2004. 


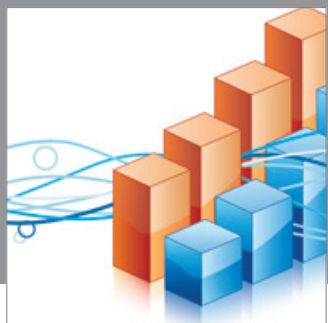

Advances in

Operations Research

mansans

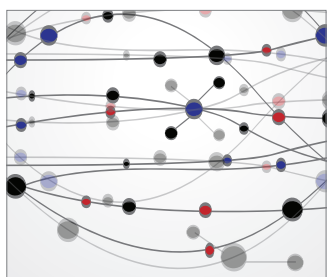

The Scientific World Journal
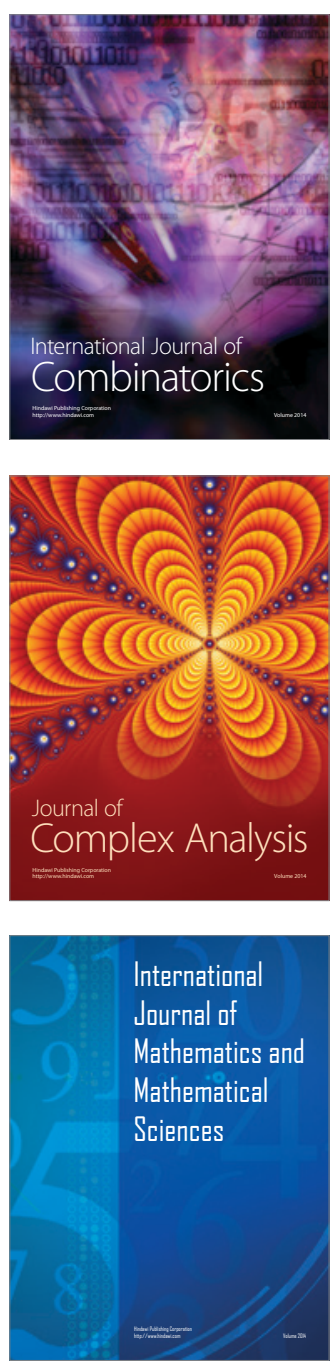
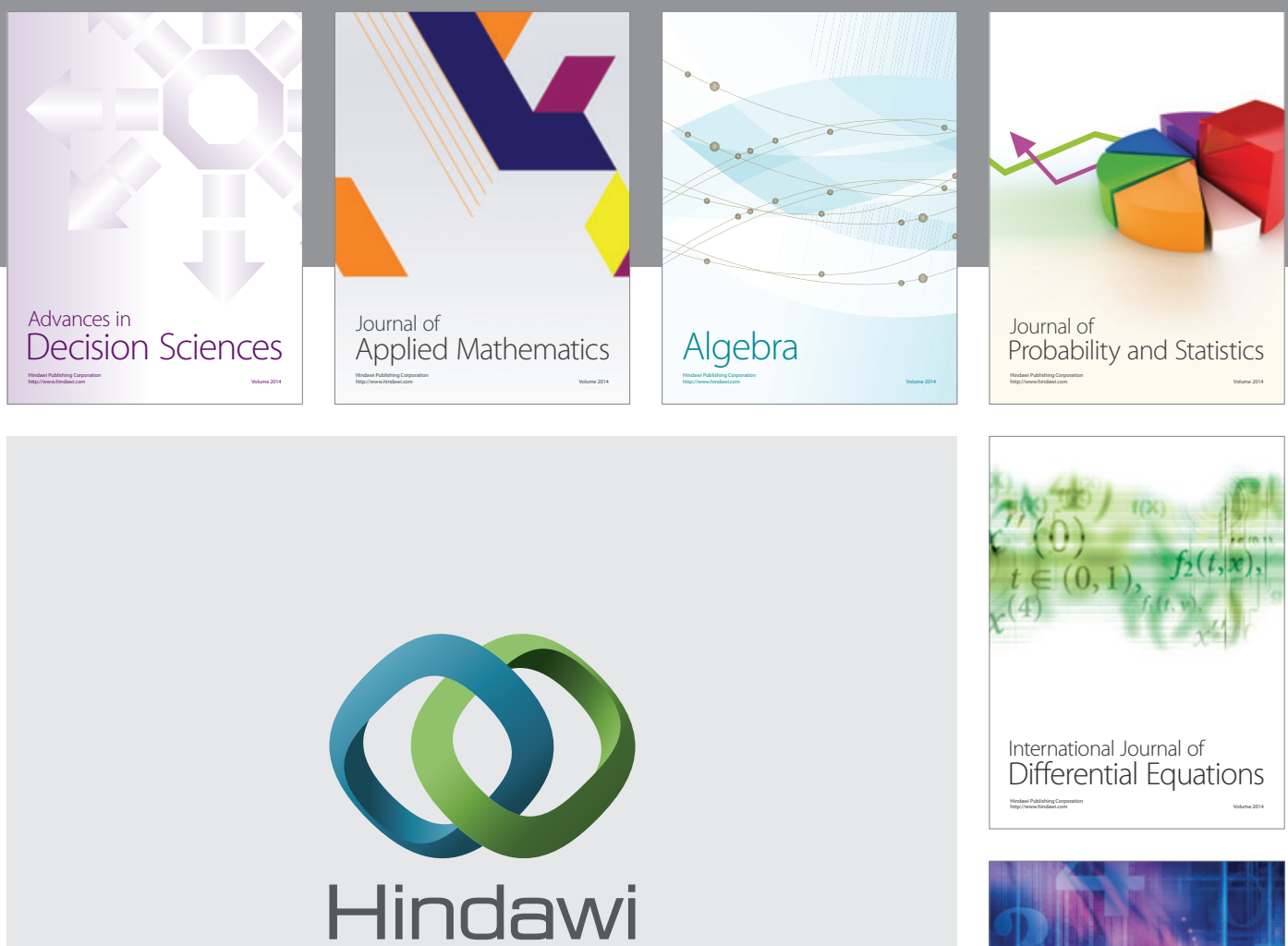

Submit your manuscripts at http://www.hindawi.com
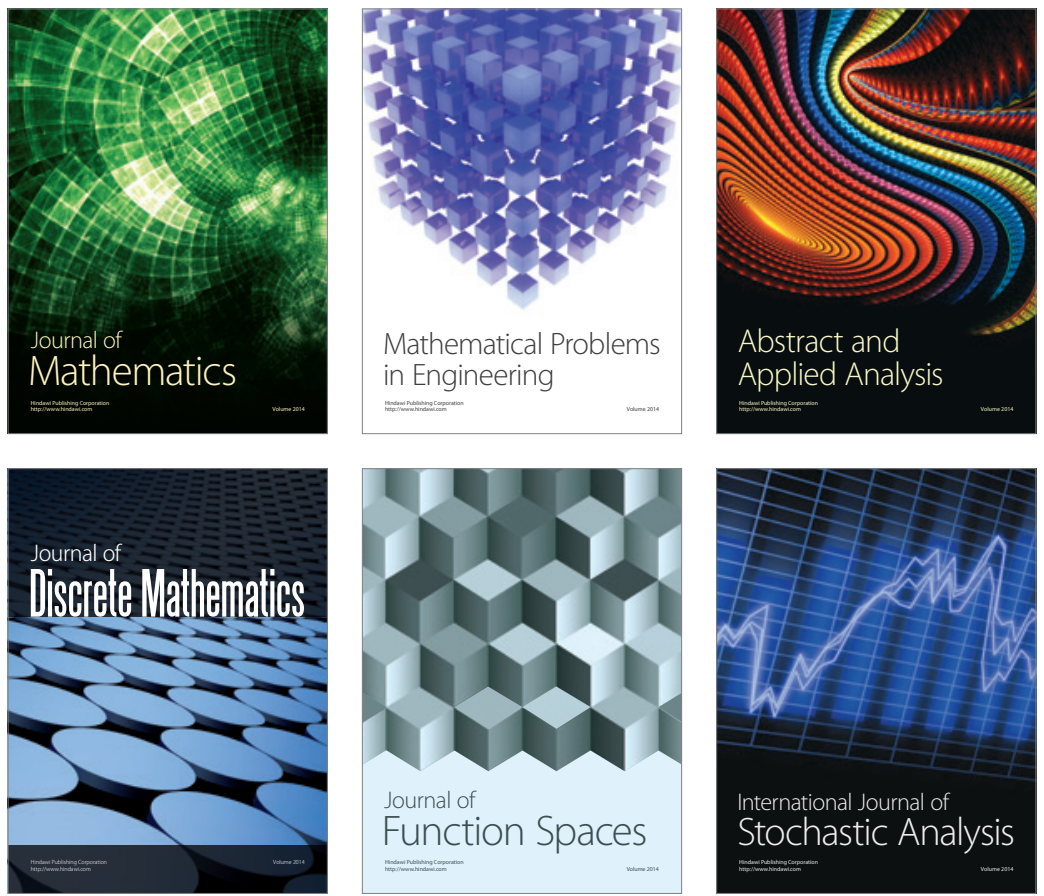

Journal of

Function Spaces

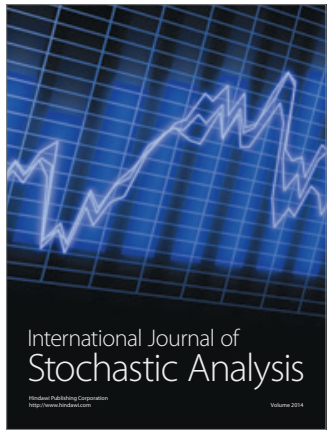

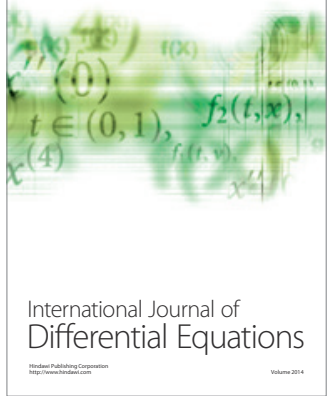
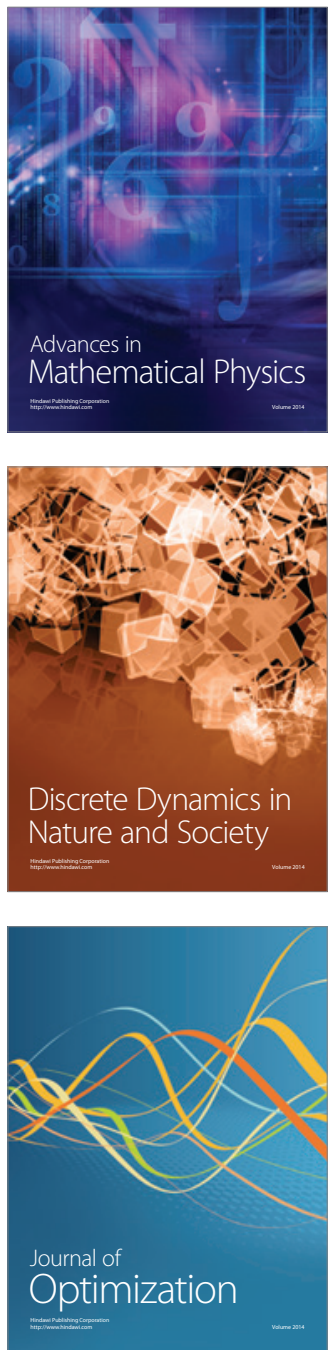